\title{
CONTAMINAÇÃO DO SOLO COM ANTRACENO E CREOSOTO E O CRESCIMENTO VEGETAL E A COLONIZAÇÃO MICORRÍZICA PELO Glomus etunicatum ${ }^{(1)}$
}

\author{
Alessandra Monteiro de Paula ${ }^{(2)}$, José Oswaldo de Siqueira ${ }^{(3)} \&$ \\ Claúdio Roberto Fonsêca Sousa Soares ${ }^{(4)}$
}

\begin{abstract}
RESUMO
A contaminação do solo por compostos orgânicos, especialmente os hidrocarbonetos aromáticos policíclicos (HAPs) de petróleo, é um problema crescente e que traz graves conseqüências ambientais. Para avaliar os impactos causados por esses compostos, torna-se necessário conhecer seus efeitos sobre as plantas e a microbiota rizosférica associada. No presente estudo avaliaram-se os efeitos de antraceno e creosoto no crescimento e na colonização micorrízica de Brachiaria brizantha e Pueraria phaseoloides. Antraceno e creosoto foram aplicados a um solo infestado com o fungo micorrízico Glomus etunicatum, sendo: antraceno nas concentrações de $0 ; 0,25 ; 0,5 ; 0,75$ e $1 \mathrm{~g} \mathrm{~kg}^{-1}$ solo; e creosoto nas concentrações de $0 ; 0,5 ; 1 ; 2 \mathrm{e} 3 \mathrm{~g} \mathrm{~kg}^{-1}$ solo. $O$ solo com os tratamentos foi colocado em tubetes $\left(290 \mathrm{~cm}^{3}\right)$ e semeado com as plantas-teste, as quais foram cultivadas por seis semanas. Verificou-se que o antraceno não afetou o crescimento da puerária e teve pequeno estímulo no crescimento da braquiária na dose mais baixa, enquanto o creosoto reduziu o crescimento da braquiária e não teve efeito na puerária. Entretanto, ambos os contaminantes inibiram a colonização micorrízica da puerária, atingindo redução de cerca de $90 \%$ em relação ao controle. Em concentrações bem inferiores às encontradas em solos contaminados, a colonização micorrízica foi inibida em 50 \%, ficando evidente o potencial de impacto desses poluentes na relação planta-fungo micorrízico. Na braquiária não foi encontrada colonização micorrízica. Fica evidenciada a resposta diferenciada das duas espécies
\end{abstract}

\footnotetext{
(1) Projeto financiado pelo CNPq. Extraído da Tese de Mestrado do primeiro autor, apresentada ao Programa de Pós-Graduação em Solos e Nutrição de Plantas, Universidade Federal de Lavras - UFLA. Recebido para publicação em dezembro de 2005 e aprovado em abril de 2007.

(2) Doutoranda do Programa de Pós-Graduação em Solos e Nutrição de Plantas, Laboratório de Microbiologia do Solo, Escola Superior de Agricultura "Luiz de Queiroz" - ESALQ/USP. Caixa Postal 09, CEP 13418-900 Piracicaba (SP). Bolsista CAPES. Email: ampaula@esalq.usp.br

(3) Professor Titular do Departamento de Ciência do Solo, Universidade Federal de Lavras - UFLA. Caixa Postal 3037, CEP 37200000 Lavras (MG). Bolsista do CNPq. E-mail: siqueira@ufla.br

${ }^{(4)}$ Assistente de Pesquisa do Departamento de Ciência do Solo, UFLA. Bolsista Prodoc-CAPES, E-mail: crfsoares@gmail.com
} 
estudadas aos contaminantes e o acentuado efeito negativo destes sobre a colonização micorrízica. A puerária, por ter se mostrado insensível aos HAPs, nas concentrações estudadas, apresenta potencial para aplicação na fitorremediação de áreas impactadas por esses contaminantes.

Termos de indexação: HAPs, fungos do solo, micorrizas arbusculares, fitorremediação.

\title{
SUMMARY: SOIL CONTAMINATION WITH ANTHRACENE AND CREOSOTE: IMPACT ON PLANT GROWTH AND MYCORRHIZAL COLONIZATION BY Glomus etunicatum
}

\begin{abstract}
Soil contamination by organic compounds, especially by the petroleum-derived, polycyclic aromatic hydrocarbons $\left(P A H_{s}\right)$, is a growing problem with serious environmental consequences. To evaluate the environmental impact of these products it is important to understand their effects on plants and the associated microbiota. The effects of PAHS on growth and mycorrhizal colonization of Brachiaria brizantha and Pueraria phaseoloides were evaluated here. Two PAHs, anthracene and creosote, were applied to a soil infested with the mycorrhizal fungus Glomus etunicatum at varied concentrations: anthracene ( 0 ; 0.25 ; $0.5 ; 0.75$ and $1 \mathrm{~g} \mathrm{~kg}^{-1}$ soil) and creosote (0; 0.5; $1 ; 2$ and $3 \mathrm{~g} \mathrm{~kg}^{-1}$ soil). This soil was packed into plastic tubes $\left(290 \mathrm{~cm}^{3}\right)$ where test plants were sown and grown for six weeks. It was found that anthracene did not affect pueraria growth and had a slight stimulus on brachiaria growth at the lowest concentration, whereas creosote had no effect on pueraria either, but inhibited brachiaria growth. Both contaminants inhibited mycorrhizal colonization in pueraria by about $90 \%$, compared to the control. At concentrations below those found in contaminated soils, AM colonization was inhibited by $50 \%$. The potential impact of these products on plant-AM fungus relationships is quite evident here. No colonization was found in brachiaria, regardless of the presence of PAHs. The results showed a differentiated sensitivity of the plants to the contaminants and their marked negative effect on $\mathrm{G}$. etunicatum root colonization. In the evaluated concentration range, pueraria was insensitive to both compounds, which indicates the species for further studies on phytoremediation of areas under the impact of these contaminants.
\end{abstract}

Index terms: PAHs, soil fungi, arbuscular mycorrhiza, phytoremediation.

\section{INTRODUÇÃO}

A intensificação das atividades industriais e agrícolas, em conseqüência do desenvolvimento tecnológico e do crescimento populacional nas últimas décadas, tem ocasionado aumento dos níveis de contaminantes orgânicos no solo. Os poluentes orgânicos derivados de petróleo e do coque destacam-se dentre os demais contaminantes, devido ao grande volume de produção, industrialização, transporte e usos diversos dos seus derivados (Schwab \& Banks, 1999).

Dentre os poluentes orgânicos de solo mais comuns, destacam-se o creosoto e o antraceno. O creosoto é um óleo derivado da destilação do coque amplamente utilizado na preservação da madeira e formado por uma mistura de hidrocarbonetos aromáticos policíclicos (HAPs) e monocíclicos líquidos e sólidos (85\%), compostos fenólicos (10\%) e compostos S-, N- e O- heterocíclicos (5\%). Este poluente possui elevada capacidade de inibição dos fungos (Muncnerova \& Augustin, 1994; Richter et al.,
2003) e tem efeitos mutagênicos e carcinogênicos sobre outros organismos. A contaminação do solo por creosoto é muito freqüente e ocorre devido a vazamentos de tanques, gotejamento de madeiras tratadas, derramamentos e pela lixívia de lagos artificiais de contenção (Mueller et al., 1989), podendo contaminar o solo, as águas superficiais e o lençol freático. A composição complexa deste poluente representa um desafio para técnicas de biorremediação de ambientes contaminados (Mueller et al., 1989). Outros poluentes importantes derivados de petróleo apresentam várias ligações benzeno condensadas, sendo na maioria não-voláteis, representados por mais de 100 diferentes compostos, como o antraceno, que é formado por três anéis aromáticos fundidos (Prince \& Drake, 1999). Em razão das suas características químicas, o antraceno e outros HAPs são relativamente resistentes à biodegradação, podendo se acumular em concentrações elevadas no ambiente, havendo a necessidade de intervenção humana para acelerar a dissipação desses compostos em ambientes contaminados. 
Solos contaminados com HAPs têm sido tratados por biorremediação (Prince \& Drake, 1999) e estudos recentes indicam que a presença de plantas acelera $\mathrm{e}$ aumenta a degradação desses compostos (Susarla et al., 2002; Merkl et al., 2005). As modificações físicoquímicas e nutricionais estimulam os microrganismos decompositores que ocorrem na rizosfera, facilitando a degradação dos contaminantes (Reilley et al., 1996). As espécies vegetais variam muito quanto a esta capacidade e quanto à adaptação ao ambiente contaminado (Cunningham et al., 1996). Huang et al. (2004a) demonstraram que a combinação de landfarming, bioaumento e fitorremediação incrementou os processos metabólicos no solo, aumentando a dissipação de creosoto. Pradhan et al. (1998) demonstraram que componentes carcinogênicos dos HAPs tiveram maior dissipação na presença da gramínea Panicum sp. Segundo Kim et al. (2004), os exsudatos radiculares são capazes de solubilizar de 25 a $80 \%$ do antraceno presente no solo, e isso pode contribuir para a ação biodegradadora dos microrganismos heterotróficos na rizosfera. Em estudo recente, Kirk et al. (2005) verificaram que Lolium perenne e Medicago sativa são capazes de alterar a estrutura da comunidade microbiana na rizosfera e aumentar a população de bactérias degradadoras de hidrocarbonetos em solo contaminado com petróleo, podendo assim ampliar o potencial biorremediador do sistema. Entretanto, não se conhecem estudos dessa natureza para os fungos rizosféricos.

Sabe-se que a grande maioria das plantas encontrase, em condições naturais, invariavelmente associadas a fungos mutualistas em suas raízes, formando as micorrizas arbusculares (MAs). Essa simbiose pode exercer efeito protetor sobre as plantas por meio da melhoria do estado nutricional ou redução do efeito tóxico de compostos orgânicos (Siqueira et al., 1991; Binet el al., 2000). Plantas micorrizadas apresentam maior sistema radicular e maior capacidade absortiva em virtude da extensão do micélio extra-radicular. Isso facilita a absorção de íons e de água do solo, aumentando a ação filtrante e purificadora deste (Safir et al., 1990). Apesar de as MAs já serem estudadas mesmo em áreas degradadas, são raros os estudos em solos com poluentes orgânicos que podem exercer forte ação inibitória sobre os fungos micorrízicos arbusculares (FMAs) (Joner \& Leyval, 2003). Esses efeitos sobre os FMAs podem inibir a colonização micorrízica, o que aumentaria a susceptibilidade das plantas à ação tóxica desses contaminantes e afetaria o potencial remediador destas, conforme demonstrado por Joner et al. (2001) para Trifolium repens e Lolium perenne em solo contaminado artificialmente com HAPs de petróleo.

Estudos dos efeitos de HAPs sobre os FMAs em solos brasileiros são inexistentes e, como o País é um grande produtor e consumidor de petróleo e derivados, gerando grande quantidade de resíduos poluentes, torna-se necessário conhecer os efeitos desses contaminantes sobre as plantas e seus associados e desenvolver técnicas eficientes de remediação. No presente trabalho são relatados os efeitos da contaminação do solo com dois HAPs, creosoto e antraceno, sobre o crescimento e a colonização micorrízica de Brachiaria brizantha e Pueraria phaseoloides em condições controladas.

\section{MATERIAL E MÉTODOS}

Um estudo composto de dois ensaios foi realizado em casa de vegetação, no Departamento de Ciência do Solo da Universidade Federal de Lavras (UFLA), em Lavras-MG, para avaliar o efeito de antraceno e creosoto no crescimento e na colonização micorrízica de $B$. brizantha e $P$. phaseoloides, durante o período de seis semanas, entre setembro e novembro de 2004. O delineamento experimental utilizado foi o inteiramente casualizado, em esquema fatorial $5 \times 2$, compreendendo cinco doses de cada contaminante e as duas espécies vegetais, todas inoculadas com FMAs, totalizando 10 tratamentos, com cinco repetições. Empregou-se uma amostra de um Latossolo VermelhoAmarelo distrófico típico (LVd) coletado em área de mata no campus da UFLA. O material de solo foi seco, peneirado (4 mm) e submetido à correção do solo, aplicando-se calcário dolomítico (PRNT $100 \%$ ) para elevar o índice de saturação por bases para $60 \%$, quando o solo apresentou: $\mathrm{pH}$ (água) $=6,4 ; \mathrm{V}(\%)=$ $70 \%$; matéria orgânica $=34 \mathrm{~g} \mathrm{~kg}^{-1} ; 2,5$ e $30 \mathrm{mg} \mathrm{dm}^{-3}$ de $\mathrm{P}$ e K; 7,4, 0,7, 68, 3,7, 1,4, 0,6 e 8,4 $\mathrm{mg} \mathrm{dm}^{-3}$ de $\mathrm{Na}, \mathrm{Zn}, \mathrm{Fe}, \mathrm{Mn}, \mathrm{Cu}, \mathrm{B}$ e S, respectivamente; e 3,4, 1,9 e $2,3 \mathrm{cmol}_{\mathrm{c}} \mathrm{dm}^{-3} \mathrm{de} \mathrm{Ca}^{2+}, \mathrm{Mg}^{2+}$ e $\mathrm{H}+\mathrm{Al}$, respectivamente. Fósforo, Ke micronutrientes foram extraídos por Mehlich-1; $\mathrm{Ca}^{2+} \mathrm{e} \mathrm{Mg}^{2+}$, por $\mathrm{KCl} 1 \mathrm{~mol} \mathrm{~L}^{-1}$; $\mathrm{H}+\mathrm{Al}$, pelo método do tampão SMP; e a matéria orgânica, por oxidação com dicromato de sódio, conforme Embrapa (1997). Após a incubação, o solo foi fumigado com a aplicação de brometo de metila $98 \%$ (brometo de metila $98 \%$ + cloropicrina $2 \%$ ) na dose de $393 \mathrm{~cm}^{3} \mathrm{~m}^{-3}$, a fim de eliminar propágulos de fungos micorrízicos arbusculares (FMAs) indígenas. Antes da semeadura foi realizada uma adubação com NPK (4-14-8), sendo aplicados $4 \mathrm{mg} \mathrm{kg}^{-1}$ solo de N, $7 \mathrm{mg} \mathrm{kg}^{-1}$ solo de $\mathrm{P}$ e $6,7 \mathrm{mg} \mathrm{kg}^{-1}$ solo de K. O solo corrigido foi colocado em tubetes de polietileno de $290 \mathrm{~cm}^{3}$, nos quais foram aplicados os tratamentos e realizada a inoculação com FMAs. Para inoculação, empregaram-se propágulos de Glomus etunicatum Gedermann \& Nicolson obtidos de vasos de multiplicação em Brachiaria decumbens Stapf. da coleção do Laboratório de Microbiologia do Solo da UFLA, o qual foi empregado em razão da sua comum ocorrência em vários ecossistemas brasileiros (Stürmer $\&$ Siqueira, 2006). No momento da semeadura foram aplicados $3 \mathrm{~mL}$ de solo-inóculo por tubete, contendo fragmentos de raízes colonizadas, hifas do solo e aproximadamente 100 esporos $\mathrm{dm}^{-3}$ de solo. As sementes de $B$. brizantha e $P$. phaseoloides foram 
escarificadas com ácido sulfúrico concentrado (10 e 1 min, respectivamente). As sementes de $P$. phaseoloides foram também inoculadas com a estirpe de rizóbio SEMIA 6175 (BR 2613). Foram semeadas de três a cinco sementes, procedendo-se ao desbaste para uma planta por tubete, 15 dias após a semeadura.

No ensaio com antraceno foram aplicadas cinco doses do produto (Sigma $97 \%$ ): 0; 0,25; 0,50; 0,75 e $1 \mathrm{~g} \mathrm{~kg}^{-1}$ de solo, definidas com base no estudo de Joner \& Leyval (2001). Para aplicação, o produto foi dissolvido em álcool absoluto e adicionado ao solo, o qual foi misturado e incubado por 15 dias antes da semeadura. Após a adição do antraceno, adicionaramse $50 \mathrm{~mL} \mathrm{~kg}{ }^{-1}$ solo de uma suspensão de solo $\left(50 \mathrm{~cm}^{-3}\right.$ de solo em $1 \mathrm{~L}$ de água), visando reintroduzir os microrganismos nativos, exceto FMAs. No ensaio com creosoto, cinco doses de Palum ${ }^{\circledR}$ (Eurofarma Laboratórios Ltda. - 100 \% óleo de creosoto) foram aplicadas ao solo nas concentrações de 0; 0,5; $1 ; 2 \mathrm{e}$ $3 \mathrm{~g} \mathrm{~kg}^{-1}$ de solo, sendo estas concentrações baseadas em estudo realizado por Huang et al. (2004a). O produto foi aplicado diretamente no solo e incubado por um período de 15 dias. Demais procedimentos foram realizados conforme descrito para o primeiro ensaio.

Nos dois ensaios, as plantas foram mantidas em casa de vegetação por seis semanas com umidade adequada, controlada por irrigações diárias. No final do período de crescimento, as plantas foram retiradas dos tubetes e separadas em raízes e parte aérea. A parte aérea foi seca em estufa com circulação de ar a $65{ }^{\circ} \mathrm{C}$ (até peso constante), para determinação da matéria seca. Para avaliação da colonização micorrízica, aproximadamente $1 \mathrm{~g}$ de raízes frescas foi separado para coloração, conforme Phillipis \& Haymann (1970), e avaliado quanto à percentagem de raiz colonizada, segundo Giovannetii \& Mosse (1980).

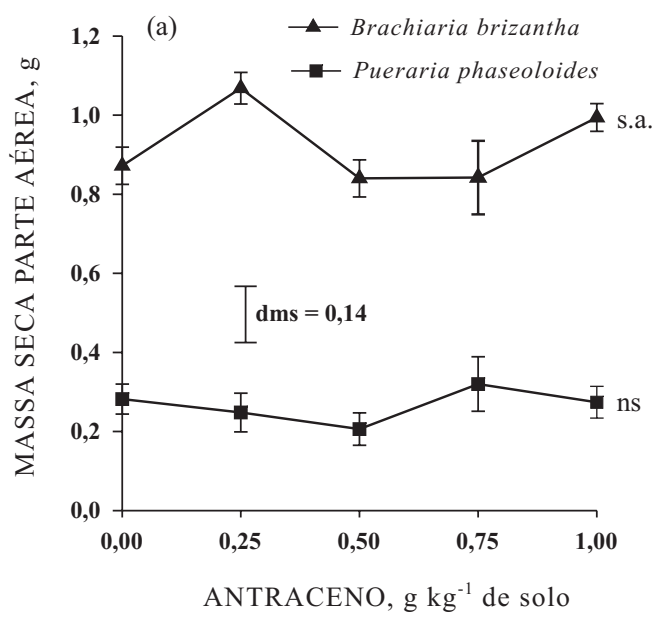

Os resultados foram submetidos à análise de variância por meio do programa estatístico SISVAR (Ferreira, 2000). Curvas de regressão foram ajustadas pelo programa TableCurve 2D for Windows v.3.01 (Jandel Corp.).

\section{RESULTADOS E DISCUSSÃO}

A aplicação do antraceno em baixa concentração $\left(0,25 \mathrm{~g} \mathrm{~kg}^{-1}\right.$ solo) exerceu estímulo em $B$. brizantha e não teve efeito sobre $P$. phaseoloides (Figura 1a). $\mathrm{O}$ efeito estimulante de derivados de petróleo sobre as plantas já foi observado por Maliszewska-Kordybach \& Smreczak (2000), trabalhando com baixas concentrações de antraceno $\left(0,1 \mathrm{~g} \mathrm{~kg}^{-1}\right.$ de solo). Eles verificaram ainda que concentrações de até $20 \mathrm{mg} \mathrm{kg}^{-1}$ de fluoreno, antraceno, pireno e criseno no solo não inibiram o crescimento de tomate, feijão, trigo e milho e, ao contrário, estimularam o crescimento de aveia e girassol. Os derivados de petróleo são hidrofóbicos e podem se ligar aos componentes orgânicos do solo (Johnsen et al., 2005), reduzindo a biodisponibilidade na solução e o efeito tóxico para as plantas. Como o solo empregado no presente estudo apresentava $34 \mathrm{~g} \mathrm{~kg}^{-1}$ de matéria orgânica, é provável que isso tenha contribuído para a baixa fitotoxicidade do antraceno. Entretanto, este hidrocarboneto exerce efeito fitotóxico quando presente em concentrações mais elevadas no solo $\left(5,9 \mathrm{~g} \mathrm{~kg}^{-1}\right)$, como constatado por Baund-Grasset et al. (1993).

O aumento da concentração de creosoto no solo teve efeito diferenciado no crescimento das duas espécies (Figura 1b). Tal como verificado no ensaio com antraceno, $P$. phaseoloides foi insensível às concentrações de creosoto estudadas, enquanto $B$. brizantha teve o crescimento reduzido com a aplicação

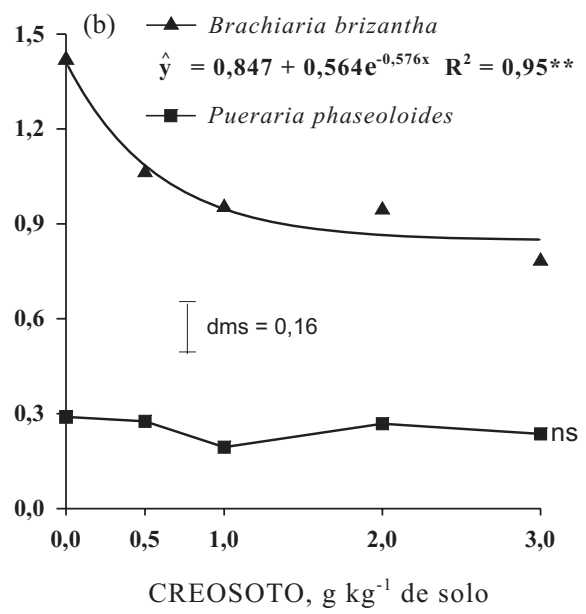

Figura 1. Matéria seca da parte aérea de B. brizantha e $P$. phaseoloides inoculada com G. etunicatum em solo tratado com antraceno (a) e creosoto (b); ** efeito significativo a $5 \%$; ns - diferença não-significativa entre as doses; s.a. - sem ajuste polinomial. 
deste. A inibição em $B$. brizantha ocorreu já na primeira dose aplicada ( $0,5 \mathrm{~g} \mathrm{~kg}^{-1}$ de solo), indicando elevada sensibilidade desta espécie ao produto, sendo verificada redução de 30 \% na matéria seca da parte aérea na dose de $1 \mathrm{~g} \mathrm{~kg}^{-1}$ de solo, em relação ao controle. Esse comportamento de $B$. brizantha corrobora os resultados de Huang et al. (2004b), que constataram efeito adverso da contaminação do solo com creosoto em três gramíneas, o qual promoveu decréscimo de $50 \%$ no crescimento de Elymus canadensis na dose de $1 \mathrm{~g} \mathrm{~kg}^{-1}$ de solo; para as espécies Festuca arundinacea e Poa pratensis essa redução ocorreu apenas na maior dose ( $3 \mathrm{~g} \mathrm{~kg}^{-1}$ de solo). Os resultados aqui relatados também estão de acordo com os de Hernández-Valencia \& Mager (2003), que observaram, no México, efeito negativo de hidrocarbonetos de petróleo em $B$. brizantha. Segundo esses autores, concentrações de até $30 \mathrm{~g} \mathrm{~kg}^{-1}$ de solo de hidrocarbonetos poluidores não limitaram o crescimento das plantas nos primeiros 45 dias de exposição, mas causaram redução acentuada aos 280 dias. Esses resultados indicam que B. brizantha apresenta sensibilidade aos hidrocarbonetos derivados de petróleo, ao contrário de $P$. phaseoloides.

Tanto o antraceno como o creosoto exerceram efeito inibitório acentuado na colonização micorrízica de $P$. phaseoloides (Figura 2). Verificou-se que a redução na colonização ocorreu já nas concentrações mais baixas de antraceno e creosoto, sendo esse efeito inibitório acentuado com a elevação das doses, ficando evidente o efeito negativo desses compostos sobre a colonização de G. etunicatum em P. phaseoloides. A dose de antraceno capaz de reduzir em $50 \%$ a colonização micorrízica foi estimada em apenas $0,30 \mathrm{~g} \mathrm{~kg}^{-1}$ de solo, indicando que este composto, mesmo em concentrações mais baixas em relação ao que pode ser encontrado em solos próximos a regiões industriais, em geral acima de $1 \mathrm{~g} \mathrm{~kg}^{-1}$ de solo (WHO, 1998), exerce efeito inibitório acentuado na colonização micorrízica de $P$. phaseoloides. Isso, além de limitar o crescimento das plantas, pode agravar os impactos desses contaminantes nos ecossistemas terrestres, pois as MAs desempenham importante papel na funcionalidade destes (Koide, 2000). Esses resultados evidenciam efeitos distintos deste poluente na planta e na micorrização, não exercendo efeito fitotóxico para $P$. phaseoloides, porém causando grande impacto na relação desta espécie com o fungo G. etunicatum. A elevada sensibilidade da colonização micorrízica ao creosoto manifestou-se já na primeira dose $\left(0,5 \mathrm{~g} \mathrm{~kg}^{-1}\right.$ de solo), sendo a dose capaz de reduzir em $50 \%$ a colonização micorrízica estimada em $0,47 \mathrm{~g} \mathrm{~kg}^{-1} \mathrm{de}$ solo. Não foram encontrados estudos que avaliam o efeito de creosoto sobre a colonização por FMAs em solos tropicais, sendo este o primeiro relato sobre esse tema no Brasil. A sensibilidade das MAs a estes compostos está em concordância com Gaspar et al. (2002), que constataram redução de $50 \%$ na colonização micorrízica do milho por Glomus geosporum, isolado de área contaminada com hidrocarbonetos, na presença de $0,1 \mathrm{~g} \mathrm{~kg}^{-1}$ de fenantreno. Joner \& Leyval (2001) também verificaram acentuado efeito negativo da contaminação do solo com $500+500+50 \mathrm{mg} \mathrm{kg}^{-1}$ de antraceno, criseno e dibenzo(a,h) antraceno no solo na colonização micorrízica do trevo (Trifolium repens) e azevém (Lolium perenne) cultivados juntos. Binet et al. (2000), avaliando o efeito da contaminação com antraceno na concentração de $5 \mathrm{~g} \mathrm{~kg}^{-1}$ de solo, verificaram também que a adição do contaminante não afetou o peso da parte aérea seca do azevém (Lolium perenne), independentemente da inoculação com Glomus mosseae. Os autores atribuíram esse resultado à procedência do fungo, que havia sido isolado de uma área contaminada e, portanto, estaria adaptado aos contaminantes. Além disso, os autores encontraram menor quantidade de antraceno transportada para a parte aérea nas plantas (a)

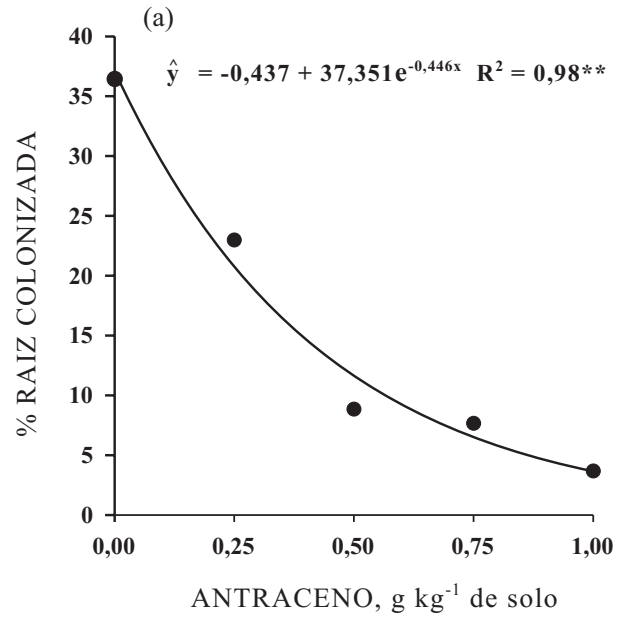

(b)

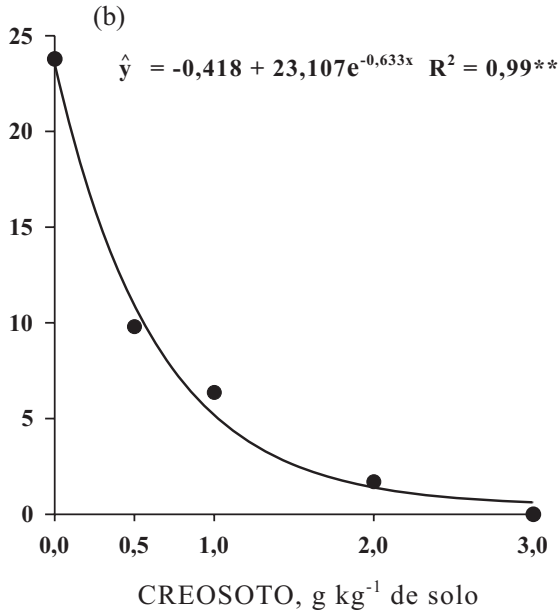

Figura 2. Colonização micorrízica de $P$. phaseoloides inoculada com $G$. etunicatum em solo tratado com doses crescentes de antraceno (a) e creosoto (b). ** efeito significativo a $5 \%$. 
micorrizadas, indicando ação protetora e possibilidade de sobrevivência em ambientes contaminados.

No presente estudo não foi observada colonização micorrízica de $B$. brizantha por $G$. etunicatum e, portanto, o efeito dos contaminantes sobre esta espécie não pôde ser avaliado. A ausência de colonização micorrízica em $B$. brizantha neste experimento não pôde ser explicada, pois esta espécie tem se mostrado compatível com G. etunicatum (Santos et al., 2001) e o inóculo empregado era viável, uma vez que ele colonizou satisfatoriamente $P$. phaseoloides nas mesmas condições.

Apesar de existirem relatos sobre os benefícios das MAs sobre o crescimento das plantas em ambientes contaminados com hidrocarbonetos de petróleo (Cabello, 1999; Joner \& Leyval, 2001), os efeitos desses compostos na relação planta-fungo são ainda desconhecidos. Os propágulos de FMAs podem sobreviver em ambientes contaminados com hidrocarbonetos de petróleo (Cabello, 1997; Paula et al., 2006), mas sua eficiência na colonização e os efeitos sobre as plantas hospedeiras ainda são pouco estudados. Os resultados aqui apresentados indicam a alta sensibilidade da colonização micorrízica aos HAPs de petróleo e que, como os fungos que formam as MAs são componentes essenciais dos ecossistemas, esses efeitos sobre as raízes e suas funções podem ser de grande relevância para o impacto ambiental dos HAPs e na remediação dessas áreas.

\section{CONCLUSÕES}

1. Pueraria phaseoloides mostrou-se insensível ao antraceno e creosoto, enquanto Brachiaria brizantha foi também insensível ao antraceno, porém sensível ao creosoto nas condições e doses aplicadas no solo.

2. Ao contrário do efeito observado no crescimento das plantas, os dois HAPs estudados exercem efeito inibitório acentuado na colonização de $P$. phaseoloides por Glomus etunicatum.

\section{LITERATURA CITADA}

BAUND-GRASSET, F.; BAUND-GRASSET, S. \& SAFFERMAN, S.I. Evaluation of a contaminated soil with phytotoxicity tests. Chemosphere, 26:1365-1374, 1993.

BINET, P.; PORTAL, J. M. \& LEYVAL, C. Fate of polycyclic aromatic hydrocarbons (PAH) in the rhizosphere and mycorrhizosphere of ryegrass. Plant Soil, 227:207-213, 2000.

CABELLO, M.N. Hydrocarbon pollution: Its effects on native arbuscular mycorrhizal fungi (AMF). FEMS Microbiol. Ecol., 22:233-236, 1997.
CABELLO, M.N. Effectiveness of indigenous arbuscular mycorrhizal fungi (AMF) isolated from hydrocarbon polluted soils. J. Basic Microbiol., 39:89-95, 1999.

CUNNINGHAM, S.D.; ANDERSON, T.A.; SCHWAB, A.P. \& HSU, F. Phytoremediation of soils contaminated with organic pollutants. Adv. Agron., 56:55-114, 1996.

EMPRESA BRASILEIRA DE PESQUISA AGROPECUÁRIA EMBRAPA. Centro Nacional de Pesquisa em Solos. Manual de métodos de análise de solo. 2 ed. Rio de Janeiro, 1997. 212p.

FERREIRA, D.F. Análises estatísticas por meio do SISVAR (Sistema para análise de variância) para Windows versão 4.0. In: REUNIÃO ANUAL DA REGIÃO BRASILEIRA DA SOCIEDADE INTERNACIONAL DE BIOMETRIA, 45., São Carlos, 2000. Anais. São Carlos, Universidade Federal de São Carlos, 2000. p.255-258.

GASPAR, M.L.; CABELLO, M.N.; CAZAN, M.C. \& POLLERO, R.J. Effect of phenanthrene and Rhodotorula glutinis on arbuscular mycorrhizal fungus colonization of maize roots. Mycorrhiza, 12:55-59, 2002.

GIOVANNETTI, M. \& MOSSE, B. An evaluation of techniques for measuring vesicular arbuscular mycorrhizal infection in roots. New Phytol., 84:489-500, 1980.

HERNÁNDEZ-VALENCIA, I. \& MAGER, D. Uso de Panicum maximum y Brachiaria brizantha para fitorremediar suelos contaminados con un crudo de petróleo liviano. Bioagro, 15:149-155, 2003.

HUANG, X.; El-ALAWI, Y.; PENROSE, D.M.; GLICK, B.R. \& GREENBERG, B.M. A multi-process phytoremediation system for removal of polycyclic aromatic hydrocarbons from contaminated soils. Environ. Poll., 130:465-476, 2004a.

HUANG, X.; El-ALAWI, Y.; PENROSE, D.M.; GLICK, B.R. \& GREENBERG, B.M. Responses of three grass species to creosote during phytoremediation. Environ. Poll., 130:453-464, 2004b.

JOHNSEN, A.R.; WICK, L.Y. \& HARMS, H. Principles of microbial PAH-degradation in soil. Environ. Poll., 133:7184, 2005.

JONER, E.J.; JOHANSEN, A.; LOIBNER, A.P.; CRUZ, M.A.D.; SZOLAR, O.H.J.; PORTAL, J-M. \& LEYVAL, C. Rhizosphere effects on microbial community structure and dissipation and toxicity of polycyclic aromatic hydrocarbons (PAHs) in spiked soil. Environ. Sci. Technol., 35:2773-2777, 2001.

JONER, E.J. \& LEYVAL, C. Influence of arbuscular mycorrhiza on clover and ryegrass grown together in a soil spiked with polycyclic aromatic hydrocarbons. Mycorrhiza, 10:155-159, 2001.

JONER, E.J. \& LEYVAL, C. Phytoremediation of organic pollutants using mycorrhizal plants: A new aspect of rhizosphere interactions. Agronomie, 23:495-502, 2003.

KIM, Y.B.; PARK, K.Y.; CHUNG, Y.; OH, K.C. \& BUCHANAN, B.B. Phytoremediation of anthracene contaminated soils by different plant species. J. Plant Biol., 47:174-178, 2004. 
KIRK, J.L.; KLIRONOMOS, J.N.; LEE, H. \& TREVORS, J.T. The effects of perennial ryegrass and alfalfa on microbial abundance and diversity in petroleum contaminated soil. Environ. Poll., 133:455-465, 2005.

KOIDE, R.T. Functional complementarity in the arbuscular mycorrhizal symbiosis. New Phytol., 147:233-235, 2000.

MALISZEWSKA-KORDYBACH, B. \& SMRECZAK, B. Ecotoxicological activity of soils polluted with polycyclic aromatic hydrocarbons (PAHs) - Effect on plants. Environ. Technol., 21:1099-1110, 2000.

MERKL, N.; SCHULTZE-KRAFT, R. \& ARIAS, M. Influence of fertilizer levels on phytoremediation of crude oilcontaminated soils with the tropical pasture grass Brachiaria brizantha (Hochst. ex A. Rich.) Stapf. Inter. J. Phytor., 7:217-230, 2005.

MUELLER, J.G.; CHAPMAN, P.J. \& PRITCHARD, P.H. Creosote-contaminated sites: Their potential for bioremediation. Environ. Sci. Technol., 23:1197-1201, 1989.

MUNCNEROVA, D. \& AUGUSTIN, J. Fungal metabolism and detoxification of polycyclic aromatic-hidrocarbons A review. Biores. Technol., 48:97-106, 1994.

PAULA, A.M. ; SOARES, C.R.F.S. \& SIQUEIRA, J.O. Biomassa, atividade microbiana e fungos micorrízicos em solo de "landfarming" de resíduos petroquímicos. R. Bras. Eng. Agríc. Amb., 10:448-455, 2006.

PHILLIPS, J.M. \& HAYMAN, D.S. Improved procedures for clearing roots and staining parasitic and vesiculararbuscular mycorrhizal fungi for rapid assessment of infection. Trans. British Mycol. Soc., 55:158-161, 1970.

PRADHAN, S.P.; CONRAD, J.R.; PATEREK, J.R. \& SRIVASTAVA, V.J. Potential of phytoremediation for treatment of PAHs in soil at MGP sites. J. Soil Contam., 7:467-480, 1998.

PRINCE, R.C. \& DRAKE, E.N. Transformation and fate of polycyclic aromatic hydrocarbons in soil. In: ADRIANO, D.C.; BOLLAG, J.M.; FRANKENBERGER Jr., W.T. \& SIMS, R.C., eds. Bioremediation of contaminated soils. Madison, Soil Science Society of America, 1999. p.89-110 (Agronomy Monograph,37)
REILLEY, K.A.; BANKS, M.K. \& SCHWAB, A.P. Organic chemicals in the environment: Dissipation of polycyclic aromatic hydrocarbons in the rhizosphere. J. Environ. Qual., 25:212-219, 1996.

RICHTER, D.L.; WARNER, J.I. \& STEPHENS, A.L. A comparison of mycorrhizal and saprotrophic fungus tolerance to creosote in vitro. Inter. Biodeterior. Biodegrad., 51:195-202, 2003.

SAFIR, G.R.; SIQUEIRA, J.O.; BURTON, T.M. Vesiculararbuscular mycorrhizae in a wastewater irrigated oldfield ecosystem in Michigan. Plant Soil, 121:87-196, 1990.

SANTOS, I.P.A.; PINTO, J.C.; SIQUEIRA, J.O.; MORAIS, A.R. \& SANTOS, C.L. Influência do fósforo, micorriza e nitrogênio nos parâmetros microbiológicos da Brachiaria brizantha e Arachis pintoi consorciados. Pasturas Tropic., 23:22-26, 2001.

SCHWAB, P. \& BANKS, K. Phytoremediation of petroleumcontaminated soils. In: ADRIANO, D.C.; BOLLAG, J.M.; FRANKENBERGER Jr., W.T. \& SIMS, R.C., eds. Bioremediation of contaminated soils. Madison, Soil Science Society of America, 1999. p.783-795. (Agronomy Monograph,37)

SIQUEIRA, J.O.; SAFIR, G.R. \& NAIR, M.G. VA-mycorrhizae and mycorrhiza stimulating isoflavonoid compounds reduce plant herbicide injury. Plant Soil, 134:233-242, 1991.

STÜRMER, S.L. \& SIQUEIRA, J.O. Diversity of arbuscular mycorrhizal fungi in Brazilian ecosystems. In: MOREIRA, F.M.S.; SIQUEIRA, J.O. \& BRUSSAARD, L., eds. Soil biodiversity in Amazonian and other Brazilian ecosystems. Wallingford, CABI-Publishing, 2006. v.1. p.206-236.

SUSARLA, S.; MEDINA, V.F. \& Mc CUTCHEON, S.C. Phytoremediation: An ecological solution to organic chemical contamination. Ecol. Eng., 18:647-658, 2002.

WORLD HEALTH ORGANIZATION - WHO Environmental health criteria 202 , selected non-heterocyclic polycyclic aromatic hydrocarbons. Geneva, 1998. Disponível em: <http://www.inchem.org/documents/ehc/ehc/ ehc.202.htm>. Acessado em 17 set. 2005. 